\title{
Organization of modular networks
}

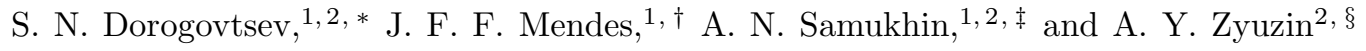 \\ ${ }^{1}$ Departamento de Física da Universidade de Aveiro, 3810-193 Aveiro, Portugal \\ ${ }^{2}$ A. F. Ioffe Physico-Technical Institute, 194021 St. Petersburg, Russia
}

\begin{abstract}
We examine the global organization of heterogeneous equilibrium networks consisting of a number of well distinguished interconnected parts - "communities" or modules. We develop an analytical approach allowing us to obtain the statistics of connected components and an intervertex distance distribution in these modular networks, and to describe their global organization and structure. In particular, we study the evolution of the intervertex distance distribution with an increasing number of interlinks connecting two infinitely large uncorrelated networks. We demonstrate that even a relatively small number of shortcuts unite the networks into one. In more precise terms, if the number of the interlinks is any finite fraction of the total number of connections, then the intervertex distance distribution approaches a delta-function peaked form, and so the network is united.
\end{abstract}

PACS numbers: 02.10.Ox, 89.20.Hh, 89.75.Fb

\section{INTRODUCTION}

Many real-world networks contain principally distinct parts with different architectures. In this sense, they are strongly heterogeneous. For example, the Internet - the net of physically interconnected computers - is connected to mobile cellular networks. One should note that the issue of the network heterogeneity is among key problems in the statistical mechanics of complex networks [1, 2, 3, 4, 5, 6, 7]. The question is how do the network's inhomogeneity influence its global structure? The quantitative description of the global organization of a network is essentially based on the statistics of $n$-th components of a vertex in the network, particularly, on the statistics of their sizes [8, 9, 10]. The $n$-th component of a vertex is defined as a set of vertices which are not farther than distance $n$ from a given vertex. From this statistics, one can easily find less informative but very useful characteristics - the distribution of intervertex distances and its first moment, the average intervertex distance. In the networks with the small-world phenomenon, socalled "small worlds", the mean length of the shortest path $\bar{\ell}(N)$ between two vertices grows slower than any positive power of the network size $N$ (the total number of vertices). Rather typically, $\bar{\ell}(N) \sim \ln N$. As a rule, in infinite small worlds, a distribution of intervertex distances approaches a delta-function form, where the mean width $\delta \ell$ is much smaller than $\bar{\ell}$. Moreover, in uncorrelated networks, $\delta \ell(N \rightarrow \infty) \rightarrow$ const. So, in simple terms, vertices in these infinite networks are almost surely mutually equidistant. This statement can be easily understood if a network has no weakly connected separate parts [11]. In this paper we consider a contrasting situation. Our

\footnotetext{
*Electronic address: sdorogov@ua.pt

${ }^{\dagger}$ Electronic address: jfmendes@ua.pt

${ }^{\ddagger}$ Electronic address: samukhin@ua.pt

§Electronic address: A.Zyuzin@mail.ioffe.ru
}

networks are divided into a number of non-overlapping but interlinked subnetworks, say $j=1,2, \ldots, m$. What is important, we suppose that the connections between these subnetworks are organized differently than inside them, see Fig. 1. This assumption results in a global (or one may say, macroscopic) heterogeneity of a network. Using the popular term "community", one can say that our networks have well distinguished communities or modules. Modular architectures of this kind lead to a variety of effects [12, 13, 14, 15]. Figure 1 explains the difference between these modular networks and the well studied $m$-partite networks [16, 17].

In this work we analytically describe the statistics of the $n$-th components in these networks when all $m$ communities are uncorrelated. For the sake of brevity, here we consider only the case of $m=2$, i.e., of two networks with shortcuts between them. As an immediate

(a)

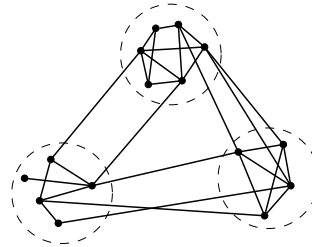

(b)

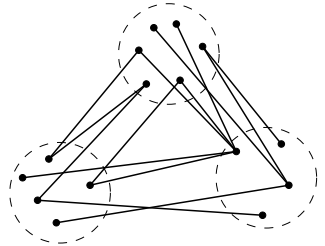

FIG. 1: (a) An example of a network, which we study in this paper in the case $m=3$. The structure of interconnections between the three non-overlapping subnetworks differs from the structure of connections inside these subnetworks. Moreover, the structures of the three subnetworks may differ. (b) A contrasting example of a 3-partite graph, where connections between vertices of the same kind are absent. 

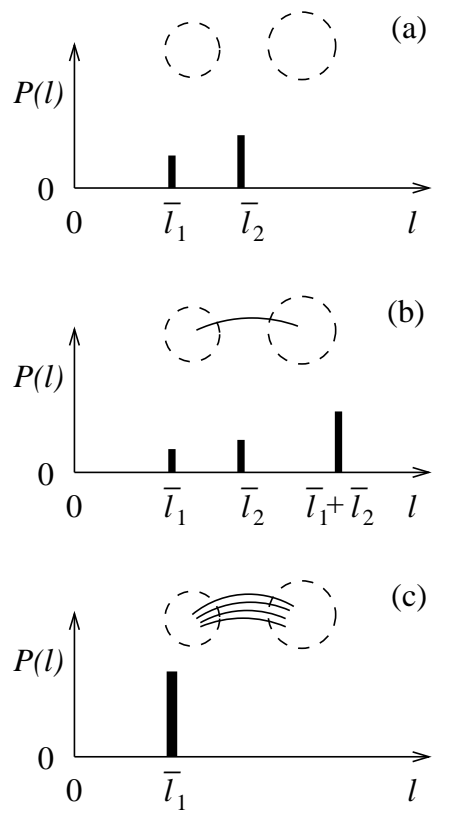

FIG. 2: Schematic view of the evolution of an intervertex distance distribution with the growing number of shortcuts between two large networks: (a) two separate networks; (b) two networks with a single shortcut between them; (c) two interconnected networks, when the number of shortcuts is a finite fraction of the number of edges in the networks. $\bar{\ell}_{1}$ and $\bar{\ell}_{2}$ are the average intervertex distances in the first and in the second networks, respectively.

application of this theory we find a distribution of intervertex distances. We show how the global architecture of this (large) network evolves with an increasing number of shortcuts, when two networks merge into one. The question is: when is the mutual equidistance property realised? How general is this feature? Figure 2 schematically presents our result. The conclusion is that the equidistance is realized when the number of shortcuts is a finite fraction of the total number of edges in the network. This finite fraction may be arbitrary small though bigger than 0 . In this respect, the large network becomes united at arbitrary small concentrations of shortcuts.

In Sec. III we briefly present our results. Section III describes our general approach to these networks based on the Z-transformation (generating function) technique. In Sec. IV we explain how to obtain the intervertex distribution for the infinitely large networks. In Sec. $\mathrm{V}$ we discuss our results. Finally, for the sake of clarity, in the Appendix we outline the Z-transformation approach in application to the configuration model of uncorrelated networks.

\section{MAIN RESULTS}

We apply the theory of Sec. III to the following problem. Two large uncorrelated networks, of $N_{1}$ and $N_{2}$ vertices, have degree distributions $\Pi_{1}(q)$ and $\Pi_{2}(q)$ with converging second moments. We assume that dead ends are absent, i.e., $\Pi_{1}(1)=\Pi_{2}(1)=0$, which guarantees that finite connected components are not essential in the infinite network limit (see Ref. 20, 21]). $\mathcal{L}$ edges interconnect randomly chosen vertices of net 1 and randomly chosen vertices of net 2 . For simplicity, we assume in this problem that $\mathcal{L}$ is much smaller than the total number of connections in the network. The question is what is the form of the intervertex distance distribution? In the infinite network limit, to describe this distribution, it is sufficiently to know three numbers: an average distance $\bar{\ell}_{1}$ between vertices of subnetwork 1 , an average distance $\bar{\ell}_{2}$ between vertices of subnetwork 2 , and an average distance $\bar{d}$ between a vertex from subnetwork 1 and a vertex from subnetwork 2. These three numbers give positions of the three peaks in the distribution. Examining the variations of these three distances with $\mathcal{L}$ one can find when the equidistance property takes place.

We introduce the following quantities:

$$
K_{1,2} \equiv \sum_{q, r} q(q-1) \Pi_{1,2}(q, r) / \bar{q}_{1,2} .
$$

Here $\Pi_{1,2}(q, r)$ are given distributions of vertices of intradegree $q$ and inter-degree $r$ in subnetworks 1,2 (see Sec. IIIfor more detail). $\bar{q}_{1}$ and $\bar{q}_{2}$ are mean interdegrees of vertices in subnetworks 1 and 2 , respectively. In terms of $K_{1}$ and $K_{2}$, the generalizations of the mean branching [ $\zeta$ in the standard configuration model, see Eqs. (A.13) in the Appendix] are

$$
\begin{gathered}
\zeta_{1}=K_{1}+\frac{\mathcal{L}^{2}}{N_{1} N_{2} \bar{q}_{1} \bar{q}_{2}} \frac{1}{\left(K_{1}-K_{2}\right) K_{1}^{2}}, \\
\zeta_{2}=K_{2}-\frac{\mathcal{L}^{2}}{N_{1} N_{2} \bar{q}_{1} \bar{q}_{2}} \frac{1}{\left(K_{1}-K_{2}\right) K_{2}^{2}} .
\end{gathered}
$$

Here we assume that $K_{1}>K_{2}$ and $\zeta_{1}>\zeta_{2}$. We also suppose that $\zeta_{1}, \zeta_{2}<\infty$. If the resulting average distance $\bar{\ell}_{1}$ between vertices of subnetwork 1 is smaller than the corresponding average distance $\bar{\ell}_{2}$ for subnetwork 2 , then we obtain asymptotically

$$
\begin{aligned}
& \bar{\ell}_{1} \cong \frac{\ln N_{1}}{\ln \zeta_{1}} \\
& \bar{\ell}_{2} \cong \bar{d}+\frac{1}{\ln \zeta_{2}} \ln \left\{N_{2}\left[\zeta_{2}^{\bar{d}}+C \mathcal{L}\right]^{-1}\right\} \\
& \bar{d} \cong \bar{\ell}_{1}+\frac{\ln \left(N_{2} / \mathcal{L}\right)}{\ln \zeta_{2}} .
\end{aligned}
$$

Here the constant $C$ is determined by the degree distributions $\Pi_{1}(q)$ and $\Pi_{2}(q)$ and is independent of $N_{1}, N_{2}$, and $\mathcal{L}$. In formula (44),$\zeta_{2}^{\bar{d}}=\left(N_{1} N_{2} / \mathcal{L}\right)^{\ln \zeta_{2} / \ln \zeta_{1}}$. Note that these asymptotic estimates ignore constant additives. Formulas (3)-(5) demonstrate that if $\mathcal{L}$ is a finite fraction of the total number of connections (in the infinite network limit), then $\bar{\ell}_{2}$ and $\bar{d}$ approach $\bar{\ell}_{1}$. The differences 
are only finite numbers. Indeed, the second terms in relations (4) and (5) are finite numbers if $N_{2} / \mathcal{L} \rightarrow$ const. [When $\mathcal{L}$ is a finite fraction of the total number of connections, $\zeta_{2}^{\bar{d}} \ll \mathcal{L}$ in Eq. (44).] On the other hand, when $\mathcal{L}$ is formally set to 1 , relation (5) gives $\bar{d}=\bar{\ell}_{1}+\bar{\ell}_{2}$, see Fig. 2(b). Furthermore, assuming $\mathcal{L} / N_{1,2} \rightarrow 0$ as $N_{1,2} \rightarrow \infty$, we have $\bar{\ell}_{2} \cong \ln N_{2} / \ln \zeta_{2}$ according to relation (4), since $\zeta_{2}^{\bar{d}}=\left(N_{1} N_{2} / \mathcal{L}\right)^{\ln \zeta_{2} / \ln \zeta_{1}} \gg \mathcal{L}$.

We also consider a special situation where subnetworks 1 and 2 are equal, so $K_{1}=K_{2} \equiv K, \bar{q}_{1}=\bar{q}_{2}$, and $N_{1}=$ $N_{2} \equiv N$. In this case the mean branching coefficients are

$$
\zeta_{1,2}=K \pm \frac{\mathcal{L}}{N \bar{q}_{1}} \frac{1}{K} .
$$

With these $\zeta_{1}$ and $\zeta_{2}$, the mean intervertex distances have the following asymptotics: $\bar{\ell}_{1}=\bar{\ell}_{2} \cong \ln N / \ln \zeta_{1}$ and $\bar{d} \cong \bar{\ell}_{1}+\ln (N / \mathcal{L}) / \ln \zeta_{2}$. Formally setting $\mathcal{L}$ to 1 we arrive at $\bar{d}=2 \bar{\ell}_{1}=2 \ell_{2}$. One should stress that all the listed results indicate a smooth crossover from two separate networks to a single united one: there is no sharp transition between these two regimes.

\section{STATISTICS OF MODULAR NETWORKS}

We consider two interlinked undirected networks, one of $N_{1}$, the other of $N_{2}$ vertices. The adjacency matrix of the joint network, $\hat{g}$, has the following structure:

$$
\hat{g}=\left[\begin{array}{cc}
\hat{g}_{1} & \hat{h} \\
\hat{h}^{T} & \hat{g}_{2}
\end{array}\right] .
$$

Here $\hat{g}_{1}=\hat{g}_{1}^{T}$ and $\hat{g}_{2}=\hat{g}_{2}^{T}$ are $N_{1} \times N_{1}$ and $N_{2} \times N_{2}$ adjacency matrices of the first and of the second subnetworks, respectively, and $\hat{h}$ is $N_{1} \times N_{2}$ matrix for interconnections. We use the following notations: latin (greek) subscripts $i, j$, etc. $\left(\alpha, \beta\right.$, etc.) take values $1,2, \ldots, N_{1}$ $\left(N_{1}+1, N_{1}+2, \ldots N_{1}+N_{2}\right)$. So, $g_{j i}, g_{\beta \alpha}, g_{j \alpha}$ and $g_{\beta i}$ are the matrix elements of $\hat{g}_{1}, \hat{g}_{2}, \hat{h}$ and $\hat{h}^{T}$, resp. We assume the whole network to be a simple one, i.e., the matrix elements of $\hat{g}$ are either 0 or 1, and the diagonal ones are all zero, $g_{i i}=g_{\alpha \alpha}=0$.

Every vertex in this network has intra-degree and inter-degree. Vertex $i$ belonging to subnetwork 1 has intra-degree $q_{i}=\sum_{j} g_{j i}$ and inter-degree $r_{i}=\sum_{\beta} g_{\beta i}$. Vertex $\alpha$ belonging to subnetwork 2 has intra-degree $q_{\alpha}=\sum_{\beta} g_{\beta \alpha}$ and inter-degree $r_{\alpha}=\sum_{j} g_{j \alpha}$. The total numbers of intra- and interlinks are $2 L_{1}=\sum_{j, i} g_{i j}$, $2 L_{2}=\sum_{\beta, \alpha} g_{\beta \alpha}$ and $\mathcal{L}=\sum_{j, \alpha} g_{j \alpha}=\sum_{\beta, i} g_{\beta i}$.

We introduce a natural generalization of the configuration model (we recommend that a reader look over Appendix to recall the configuration model and the standard analytical approach to the statistics of its components). In our random network, intralinks in subnetworks 1 and 2 are uncorrelated, and the set of interlinks connecting them is also uncorrelated. As in the configuration model, our statistical ensemble includes all possible networks with given sequences of intraand inter-degrees for both subnetworks. All the members of the ensemble are taken with the same statistical weight. Namely, there are $\mathcal{N}_{1,2}\left(N_{1}, N_{2} ; q, r\right)$ vertices in subnetworks 1,2 of intra-degree $q$ and inter-degree $r$. Here $\sum_{q, r} \mathcal{N}_{1,2}\left(N_{1}, N_{2} ; q, r\right)=N_{1,2}$. The condition $\sum_{q, r} r \mathcal{N}_{1}\left(N_{1}, N_{2} ; q, r\right)=\sum_{q, r} r \mathcal{N}_{2}\left(N_{1}, N_{2} ; q, r\right)=\mathcal{L}$, where $\mathcal{L}$ is the number of interlinks, should be fulfilled. We assume that in the thermodynamic limit $N_{1} \rightarrow \infty, N_{2} \rightarrow \infty, N_{2} / N_{1} \rightarrow \kappa<\infty$, we have $\mathcal{N}_{1,2}\left(N_{1}, N_{2} ; q, r\right) / N_{1,2} \rightarrow \Pi_{1,2}(q, r)$, where $\Pi_{1}$ and $\Pi_{2}$ are given distribution functions. Again, there is a condition that the number of edges from subnetwork 1 to 2 is the same as from 2 to 1 :

$$
\bar{r}_{1} \equiv \sum_{q, r=0}^{\infty} r \Pi_{1}(q, r)=\kappa \sum_{q, r=0}^{\infty} r \Pi_{2}(q, r) \equiv \kappa \bar{r}_{2} .
$$

Here $\bar{r}_{1,2}$ are average inter-degrees of the vertices in subnetworks 1 and 2 .

The theory of uncorrelated networks extensively uses the Z-representation (generating function) of a degree distribution:

$$
\phi(x)=\sum_{q=0}^{\infty} \Pi(q) x^{q} .
$$

Here we introduce

$$
\phi_{1,2}(x, y)=\sum_{q, r=0}^{\infty} \Pi_{1,2}(q, r) x^{q} y^{r} .
$$

In Z-representation, the average intra- $\bar{q}_{1}$ and $\bar{q}_{2}$ and inter- $\bar{r}_{1}$ and $\bar{r}_{2}$ degrees of subnetworks 1 and 2, respectively, are

$$
\bar{q}_{1,2}=\left.\frac{\partial \phi_{1,2}(x, y)}{\partial x}\right|_{x=y=1}, \bar{r}_{1,2}=\left.\frac{\partial \phi_{1,2}(x, y)}{\partial y}\right|_{x=y=1}
$$

Let $(j, i),(\beta, \alpha),(j, \alpha)$ and $(\beta, i)$ be ordered vertex pairs. Let us name their elements in the first and second position as final and initial, respectively. An end vertex degree distribution is the conditional probability for the final vertex of some (randomly chosen) ordered pair of vertices to have intra- and interdegrees $q$ and $r$, respectively, provided the vertices in this pair are connected by an edge. We have four distributions, each one depending 
on two variables:

$$
\begin{aligned}
P_{1}(q, r) & =\frac{1}{2 L_{1}} \sum_{j i}\left\langle g_{j i} \delta\left(q_{j}-1-q\right) \delta\left(r_{j}-r\right)\right\rangle, \\
P_{2}(q, r) & =\frac{1}{2 L_{2}} \sum_{\beta, \alpha}\left\langle g_{\beta \alpha} \delta\left(q_{\beta}-1-q\right) \delta\left(r_{\beta}-r\right)\right\rangle, \\
Q_{1}(q, r) & =\frac{1}{\mathcal{L}} \sum_{j, \alpha}\left\langle g_{j \alpha} \delta\left(q_{j}-q\right) \delta\left(r_{j}-1-r\right)\right\rangle \\
Q_{2}(q, r) & =\frac{1}{\mathcal{L}} \sum_{\beta, i}\left\langle g_{\beta i} \delta\left(q_{\beta}-q\right) \delta\left(r_{\beta}-1-r\right)\right\rangle .
\end{aligned}
$$

Taking into account definitions of vertex degrees, we have

$$
\begin{aligned}
& P_{1,2}(q, r)=(q+1) \Pi_{1,2}(q+1, r) / \bar{q}_{1,2}, \\
& Q_{1,2}(q, r)=(r+1) \Pi_{1,2}(q, r+1) / \bar{r}_{1,2} .
\end{aligned}
$$

In Z-representation these distribution functions take the following forms:

$$
\begin{aligned}
& \xi_{1}(x, y)=\frac{1}{2 L_{1}} \sum_{j}\left\langle q_{j} x^{q_{j}-1} y^{r_{j}}\right\rangle=\frac{1}{\bar{q}_{1}} \frac{\partial \phi_{1}(x, y)}{\partial x} \\
& \xi_{2}(x, y)=\frac{1}{2 L_{2}} \sum_{\beta}\left\langle q_{\beta} x^{q_{\beta}-1} y^{r_{\beta}}\right\rangle=\frac{1}{\bar{q}_{2}} \frac{\partial \phi_{2}(x, y)}{\partial x} \\
& \eta_{1}(x, y)=\frac{1}{\mathcal{L}} \sum_{j}\left\langle r_{j} x^{q_{i}} y^{r_{j}-1}\right\rangle=\frac{1}{\bar{r}_{1}} \frac{\partial \phi_{1}(x, y)}{\partial y} \\
& \eta_{2}(x, y)=\frac{1}{\mathcal{L}} \sum_{\beta}\left\langle r_{\beta} x^{q_{\beta}} y^{r_{\beta}-1}\right\rangle=\frac{1}{\bar{r}_{2}} \frac{\partial \phi_{2}(x, y)}{\partial y} .
\end{aligned}
$$

Let us introduce the $n$-th components of ordered vertex pairs, $C_{n, j i}, C_{n, \beta \alpha}, C_{n, j \alpha}$ and $C_{n, \beta i}$. These components are sets, whose elements are vertices. As is natural, the components are empty, if the vertices in a pair are not connected. The first component is either one-element set consisting of the final vertex, or empty set. For example, $C_{1, \beta i}$ is either vertex $\beta$ or $\varnothing$. The second component, if nonempty, contains also all the nearest neighbours of the final vertex, except the initial one, and so on. We have four types of the components of an edge: $C_{n, j i}$, $C_{n, \beta \alpha}, C_{n, j \alpha}$ and $C_{n, \beta i}$. They are defined in a recursive way similarly to the standard configuration model (see Appendix). Each of these four $n$-th components itself consists of two disjoint sets: one of vertices in subnetwork 1 , the other-in subnetwork 2. For example, $C_{n, j i}=$ $C_{n, j i}^{(1)} \cup C_{n, j i}^{(2)}$.

The sizes of the components are $M_{n, j i}^{(1)}=\left|C_{n, j i}^{(1)}\right|$, etc. Taking into account the locally tree-like structure of our network gives

$$
\begin{aligned}
& M_{n, j i}^{(1,2)}=g_{j i}\left[\left(\begin{array}{l}
1 \\
0
\end{array}\right)+\sum_{k \neq i} M_{n-1, k j}^{(1,2)}+\sum_{\gamma} M_{n-1, \gamma j}^{(1,2)}\right], \\
& M_{n, \beta \alpha}^{(1,2)}=g_{\beta a}\left[\left(\begin{array}{l}
0 \\
1
\end{array}\right)+\sum_{k} M_{n-1, k \beta}^{(1,2)}+\sum_{\gamma \neq \alpha} M_{n-1, \gamma \beta}^{(1,2)}\right], \\
& M_{n, j \alpha}^{(1,2)}=g_{j a}\left[\left(\begin{array}{l}
1 \\
0
\end{array}\right)+\sum_{k} M_{n-1, k j}^{(1,2)}+\sum_{\gamma \neq \alpha} M_{n-1, \gamma j}^{(1,2)} x\right], \\
& M_{n, \beta i}^{(1,2)}=g_{\beta i}\left[\left(\begin{array}{l}
0 \\
1
\end{array}\right)+\sum_{k \neq i} M_{n-1, k \beta}^{(1,2)}+\sum_{\gamma} M_{n-1, \gamma \beta}^{(1,2)}\right] .
\end{aligned}
$$

The configuration model is uncorrelated random network. So all the terms on the right-hand side of each of the four equations (14) are independent random variables. Quantities within each of two sums in these equations are equally distributed. Their statistical properties are also independent of the degree distribution of the initial vertex of the edge, i.e., of $j$ or $\beta$.

The sizes of the connected components of an edge in different networks [e.g., $M_{n, j k}^{(1)}$ and $M_{n, j k}^{(2)}$ ] are, generally, correlated. So we introduce four joint distribution functions of the component sizes in different networks. In Z-representation they are defined as follows:

$$
\begin{aligned}
& \psi_{n}^{(1)}(x, y)=\frac{1}{2 L_{1}}\left\langle\sum_{j, i} g_{j i} x^{M_{n, j i}^{(1)}} y^{M_{n, j i}^{(2)}}\right\rangle, \\
& \psi_{n}^{(2)}(x, y)=\frac{1}{2 L_{2}}\left\langle\sum_{\beta, \alpha} g_{\beta \alpha} x^{M_{n, \beta \alpha}^{(1)}} y^{M_{n, \beta \alpha}^{(2)}}\right\rangle, \\
& \theta_{n}^{(1)}(x, y)=\frac{1}{\mathcal{L}}\left\langle\sum_{j, \alpha} g_{j \alpha} x^{M_{n, j \alpha}^{(1)}} y^{M_{n, j \alpha}^{(2)}}\right\rangle, \\
& \theta_{n}^{(2)}(x, y)=\frac{1}{\mathcal{L}}\left\langle\sum_{\beta, i} g_{\beta i} x^{M_{n, \beta i}^{(1)}} y^{M_{n, \beta i}^{(2)}}\right\rangle .
\end{aligned}
$$

The recursive relations for these distributions are straightforward generalization of a relation for a usual uncorrelated network, without modularity [see Eq. A.7) in the Appendix]

$$
\begin{aligned}
& \psi_{n}^{(1)}(x, y)=x \xi_{1}\left[\psi_{n-1}^{(1)}(x, y), \theta_{n-1}^{(2)}(x, y)\right], \\
& \psi_{n}^{(2)}(x, y)=y \xi_{2}\left[\psi_{n-1}^{(2)}(x, y), \theta_{n-1}^{(1)}(x, y)\right], \\
& \theta_{n}^{(1)}(x, y)=x \eta_{1}\left[\psi_{n-1}^{(1)}(x, y), \theta_{n-1}^{(2)}(x, y)\right], \\
& \theta_{n}^{(2)}(x, y)=y \eta_{2}\left[\psi_{n-1}^{(2)}(x, y), \theta_{n-1}^{(1)}(x, y)\right] .
\end{aligned}
$$


The $n$-th component $\mathcal{C}_{n, i}\left(\mathcal{C}_{n, \alpha}\right)$ of vertex $i(\alpha)$ contains all vertices at distance $n$ from vertex $i(\alpha)$ or closer. Let $\mathcal{M}_{n, i}^{(1,2)}=\left|\mathcal{C}_{n, i}^{(1,2)}\right|$ and $\mathcal{M}_{n, \alpha}^{(1,2)}=\left|\mathcal{C}_{n, \alpha}^{(1,2)}\right|$ be sizes of the components $\left[\mathcal{C}^{(1)}\right.$ and $\mathcal{C}^{(2)}$ are the subset of $\mathcal{C}$, containing vertices of the first and second networks, respectively]. Using the locally tree-like structure of the network and absence of correlations between its vertices, we obtain the Z-transform of the joint distributions of component sizes:

$$
\begin{aligned}
\Psi_{n}^{(1)}(x, y) & =\frac{1}{N_{1}}\left\langle\sum_{i} x^{\mathcal{M}_{n, i}^{(1)}} y^{\mathcal{M}_{n, i}^{(2)}}\right\rangle \\
& =x \phi_{1}\left[\psi_{n}^{(1)}(x, y), \theta_{n}^{(1)}(x, y)\right], \\
\Psi_{n}^{(2)}(x, y) & =\frac{1}{N_{2}}\left\langle\sum_{\alpha} x^{\mathcal{M}_{n, \alpha}^{(1)}} y^{\mathcal{M}_{n, \alpha}^{(2)}}\right\rangle \\
& =y \phi_{2}\left[\psi_{n}^{(2)}(x, y), \theta_{n}^{(2)}(x, y)\right] .
\end{aligned}
$$

The conditional average sizes of the components are expressed in terms of the derivatives of the corresponding distribution functions at the point $x=y=1$. For example, the conditional average component sizes for an internal vertex pair in network 1 are expressed as follows: for the part, which belongs to the first network it is

$$
\bar{M}_{n}^{(111)}=\left\langle g_{i j} M_{n, i j}^{(1)}\right\rangle /\left\langle g_{i j}\right\rangle=\left.\frac{\partial \psi_{n}^{(1)}(x, y)}{\partial x}\right|_{x=y=1}
$$

for the component part in network 1 , and

$$
\bar{M}_{n}^{(211)}=\left\langle g_{i j} M_{n, i j}^{(2)}\right\rangle /\left\langle g_{i j}\right\rangle=\left.\frac{\partial \psi_{n}^{(1)}(x, y)}{\partial y}\right|_{x=y=1}
$$

for the part of the component, which is in network 2 . Here, (i) the first superscript index indicates whether the component is in subnetwork 1 or 2, (ii) the second superscript index indicates whether the final vertex is in subnetwork 1 or 2 , and (iii) the third superscript index indicates whether the initial vertex is in subnetwork 1 or 2. For the components of a pair with initial vertex in network 2 and final in network 1 we have:

$$
\bar{M}_{n}^{(112)}=\left\langle g_{i \alpha} M_{n, i \alpha}^{(1)}\right\rangle /\left\langle g_{i \alpha}\right\rangle=\left.\frac{\partial \theta_{n}^{(1)}(x, y)}{\partial x}\right|_{x=y=1}
$$

and

$$
\bar{M}_{n}^{(212)}=\left\langle g_{i \alpha} M_{n, i \alpha}^{(2)}\right\rangle /\left\langle g_{i \alpha}\right\rangle=\left.\frac{\partial \theta_{n}^{(1)}(x, y)}{\partial y}\right|_{x=y=1},
$$

and so on.

Using Eqs. (16) one can derive recurrent relations for the average values of $M_{n}$ and $M_{n-1}$. We introduce a pair of four-dimensional vectors:

$$
\mathbf{M}_{n}^{(1)}=\left(\begin{array}{l}
\bar{M}_{n}^{(111)} \\
\bar{M}_{n}^{(112)} \\
\bar{M}_{n}^{(121)} \\
\bar{M}_{n}^{(122)}
\end{array}\right), \quad \mathbf{M}_{n}^{(2)}=\left(\begin{array}{l}
\bar{M}_{n}^{(211)} \\
\bar{M}_{n}^{(212)} \\
\bar{M}_{n}^{(221)} \\
\bar{M}_{n}^{(222)}
\end{array}\right) .
$$

Then the recurrent relations take the forms:

$$
\mathbf{M}_{n}^{(1)}=\widehat{\boldsymbol{\zeta}} \mathbf{M}_{n-1}^{(1)}+\mathbf{m}_{1}, \quad \mathbf{M}_{n}^{(2)}=\widehat{\zeta} \mathbf{M}_{n-1}^{(2)}+\mathbf{m}_{2},
$$

where

$$
\widehat{\boldsymbol{\zeta}}=\left(\begin{array}{cccc}
\xi_{11} & 0 & \xi_{21} & 0 \\
\eta_{11} & 0 & \eta_{21} & 0 \\
0 & \eta_{22} & 0 & \eta_{12} \\
0 & \xi_{22} & 0 & \xi_{12}
\end{array}\right), \quad \mathbf{m}_{1}=\left(\begin{array}{l}
1 \\
1 \\
0 \\
0
\end{array}\right), \quad \mathbf{m}_{2}=\left(\begin{array}{l}
0 \\
0 \\
1 \\
1
\end{array}\right) .
$$

Here

$$
\xi_{\mu \nu}=\left.\partial_{\mu} \xi_{\nu}(x, y)\right|_{x=y=1}, \eta_{\mu \nu}=\left.\partial_{\mu} \eta_{\nu}(x, y)\right|_{x=y=1},
$$

$\mu, \nu=1,2$. The initial conditions are $\mathbf{M}_{1}^{(1)}=\mathbf{m}_{1}$, $\mathbf{M}_{1}^{(2)}=\mathbf{m}_{2}$. As for the average sizes of the $n$-th components of vertices, they are

$$
\begin{aligned}
\overline{\mathcal{M}}_{n}^{(11)} & =\frac{1}{N_{1}}\left\langle\sum_{i} \mathcal{M}_{n, i}^{(1)}\right\rangle=\left.\frac{\partial \Psi_{n}^{(1)}(x, y)}{\partial x}\right|_{x=y=1} \\
& =1+\bar{q}_{1} \bar{M}_{n}^{(111)}+\bar{r}_{1} \bar{M}_{n}^{(112)}, \\
\overline{\mathcal{M}}_{n}^{(21)} & =\frac{1}{N_{1}}\left\langle\sum_{i} \mathcal{M}_{n, i}^{(2)}\right\rangle=\left.\frac{\partial \Psi_{n}^{(1)}(x, y)}{\partial y}\right|_{x=y=1} \\
& =\bar{q}_{1} \bar{M}_{n}^{(211)}+\bar{r}_{1} \bar{M}_{n}^{(212)}, \\
\overline{\mathcal{M}}_{n}^{(12)} & =\frac{1}{N_{1}}\left\langle\sum_{\alpha} \mathcal{M}_{n, i}^{(1)}\right\rangle=\left.\frac{\partial \Psi_{n}^{(2)}(x, y)}{\partial x}\right|_{x=y=1} \\
& =\bar{q}_{2} \bar{M}_{n}^{(122)}+\bar{r}_{2} \bar{M}_{n}^{(121)}, \\
\overline{\mathcal{M}}_{n}^{(22)} & =\frac{1}{N_{2}}\left\langle\sum_{i} \mathcal{M}_{n, i}^{(2)}\right\rangle=\left.\frac{\partial \Psi_{n}^{(2)}(x, y)}{\partial y}\right|_{x=y=1} \\
& =1+\bar{q}_{2} \bar{M}_{n}^{(222)}+\bar{r}_{2} \bar{M}_{n}^{(221)} .
\end{aligned}
$$

Here, (i) the first superscript index of $\overline{\mathcal{M}}_{n}$ indicates whether the component is in subnetwork 1 or 2 , and (ii) the second superscript index indicates whether a mother vertex is in subnetwork 1 or 2 . Recall that $\bar{q}_{1}$ and $\bar{q}_{2}$ are the mean numbers of internal connections of vertices in subnetworks 1 and 2 , respectively; $\bar{r}_{1}$ is a mean number of connections of a vertex in subnetwork 1 , which go to subnetwork 2 ; and finally $\bar{r}_{2}$ is a mean number of connections of a vertex in subnetwork 2 , which go to subnetwork 
1. Relations (23) and (25) allow us to obtain the average sizes of all components.

Example.-Since formulas in this section are rather cumbersome, to help the readers, we present a simple demonstrative example of the application of these relations. Let us describe the emergence of a giant connected components in a symmetric situation, where both subnetworks have equal sizes and identical degree distributions $\Pi_{1,2}(q, r) \equiv \Pi(q, r)$. In this case, $\xi_{1}(x, y)=$ $\xi_{2}(x, y) \equiv \xi(x, y)$ and $\eta_{1}(x, y)=\eta_{2}(x, y) \equiv \eta(x, y)$. Also, $\psi^{(1)}(x, y)=\psi^{(2)}(x, y) \equiv \psi(x, y)$ and $\theta^{(1)}(x, y)=$ $\theta^{(2)}(x, y) \equiv \theta(x, y)$. So the relative size $S$ of a giant connected component takes the form:

$$
S=1-\phi(t, u),
$$

where $t \equiv \psi(1,1)$ and $u \equiv \theta(1,1)$ are non-trivial solutions of the equations:

$$
t=\xi(t, u), \quad u=\eta(t, u) .
$$

For example, let the subnetworks be classical random graphs, and each vertex has no interlinks with a probability $1-p$ and has a single interlink with the complimentary probability $p$. That is,

$$
\Pi(q, r)=e^{-\bar{q}} \frac{\bar{q}^{q}}{q !}\left[(1-p) \delta_{q, 0}+p \delta_{q, 1}\right],
$$

where $\bar{q}$ is the mean vertex intra-degree, so $\phi(x, y)=$ $e^{\bar{q}(x-1)}[1-p+p y]$.

For a single classical random graph with vertices of average degree $\bar{q}$, Eqs. A.15) and (A.17) give the point of the birth of a giant connected component, $\bar{q}=q_{c}=1$, and the relative size of this component $S \cong 2(\bar{q}-1)$ in the critical region.

Let us now find the birth point $\bar{q}=q_{c}(p)$ and the critical dependence $S(\bar{q}, p)$ in the modular network. For this network, we find $\xi(x, y)=\partial_{x} \phi(x, y) / \bar{q}=\phi(x, y)$ and $\eta(x, y)=\partial_{y} \phi(x, y) / \bar{r}=e^{\bar{q}(x-1)}$. Substituting these functions into Eqs. (26) and (27) directly leads to the result:

$$
q_{c}=\frac{1}{1+p}, \quad S \cong 2 \frac{(1+p)^{3}}{1+3 p}\left(\bar{q}-\frac{1}{1+p}\right),
$$

compare with a single classical random graph.

\section{INTERVERTEX DISTANCE DISTRIBUTION}

As was explained in Sec. II the intervertex distance distribution in the thermodynamic limit is completely determined by the three mean intervertex distances: $\bar{\ell}_{1}$ for subnetwork $1, \bar{\ell}_{2}$ for subnetwork 2 , and $\bar{d}$ for pairs of vertices where the first vertex is in subnetwork 1 and the second is in subnetwork 2 . The idea of the computation of these intervertex distances is very similar to that in the standard configuration model, see the Appendix,
Eq. (A.18). However, the straightforward calculations for two interconnected networks are cumbersome, so here we only indicate some points in our derivations without going into technical details.

The calculations are based on the solution of recursive relations (23). As is usual, these relations should be investigated in the range $1-x \ll 1,1-y \ll 1$ of the Z-transformation parameters. Fortunately, the problem can be essentially reduced to the calculation of two highest eigenvalues of a single $4 \times 4$ matrix. The resulting eigenvalues $\zeta_{1}$ and $\zeta_{2}$ for networks with $\mathcal{L} / N_{1,2} \ll 1$ are given by formulas (2) and (6). The $n$-th component sizes are expressed in terms of these eigenvalues. The leading contributions to the $n$-th component sizes turn out to be linear combinations of powers of the mean branchings: $A \zeta_{1}^{n}+B \zeta_{2}^{n}$. The factors $A$ and $B$ do not depend on $n$. For example, when $\zeta_{1}>\zeta_{2}$, the main contributions to $\overline{\mathcal{M}}_{n}^{(11)}$ and $\overline{\mathcal{M}}_{n}^{(21)}$ look as $\zeta_{1}^{n}+\left[\mathcal{L}^{2} /\left(N_{1} N_{2}\right)\right] \zeta_{2}^{n}$ and $\left(\mathcal{L} / N_{1}\right) \zeta_{2}^{n}$, respectively. Here we omitted non-essential factors and assumed a large $n$. This approximation is based on the tree ansatz, that is on the locally tree-like structure of the network. This ansatz works when the $n$ th components are much smaller than subnetworks 1 and 2. So the intervertex distances are obtained by comparing the sizes of relevant $n$-th components of vertices with $N_{1}$ and $N_{2}$. Since networks 1 and 2 are uncorrelated, this estimate gives only a constant additive error which is much smaller than the main contribution of the order of $\ln N_{1,2}$. (See Ref. [10] for complicated calculations beyond the tree ansatz in the standard configuration model, which allow one to obtain this constant number.)

One should emphasize an additional difficulty specific for the networks under consideration. The problem is that in some range of $N_{1}$ and $N_{2}$, and $\zeta_{1}$ and $\zeta_{2}$, while an $n$-th component in, say, network 1 is already of size $\sim N_{1}$ (failing tree ansatz), the corresponding $n$-th component in network 2 is still much smaller than $N_{2}$. In terms of Sec. III. this, e.g., means that there exists a range of $n, \bar{\ell}_{1}<n<\bar{d}$, where $\overline{\mathcal{M}}_{n}^{(11)} \sim N_{1}$ but still $\overline{\mathcal{M}}_{n}^{(21)} \ll N_{2}$. Computing $\overline{\mathcal{M}}_{n}^{(21)}$ in this regime, we use the tree ansatz, while $\overline{\mathcal{M}}_{n}^{(11)}$ is set to $N_{1}$. This approximation also produces only a constant additive error which one may ignore in these asymptotic estimates.

\section{DISCUSSION AND CONCLUSIONS}

A few points should be stressed.

(i) In Section III we derived relations for the Ztransformation of the distributions of $n$-th components. Quite similarly to the standard configuration model (see Appendix), using these formulas with $n \rightarrow \infty$ readily gives corresponding relations for the statistics of finite connected components and for the size of a giant connected component. Note that when subnetworks 1 and 2 are uncorrelated, which is our case, finite connected components are essential in an infinite network only if there 
is a finite fraction of vertices of degree 1 .

(ii) The theory of Sec. III is essentially based on the locally tree-like structure of networks under consideration. In principle one can go even beyond the tree ansatz as was done for the standard configuration model in Ref. [10]. This is a challenging problem for these networks. Since we extensively used the tree approximation in Sec. IV] our results for the intervertex distances are only asymptotic estimates.

(iii) For the sake of brevity, we obtained relations only for networks with two interlinked subnetworks, but it is not a restriction. A generalization to networks with an arbitrary number of interlinked subnetworks is straightforward. The final relations in Sec. III can be readily generalized without derivation. Generalization to structured networks with degree-degree correlations is also clear. Note that, in particular, our theory can describe multi-partite networks, whose subnetworks have no intraconnections. Based on equations derived for the configuration model 22, 23] (for $k$-cores in real-world networks, see Refs. 24, 25]), one can also generalize this theory to describe the $k$-core organization of modular networks

(iv) As an application, we considered interlinked networks with a relatively small number of shortcuts. Note however that our general results in Sec. [II]do not assume this restriction.

In summary, we have developed an analytical approach to the statistics of networks with well distinguished communities. We have derived general relations allowing one to find the distributions of the sizes of connected components in these networks. As a particular application of this theory, we have obtained asymptotic estimates for the distribution of intervertex distances in two weakly interconnected uncorrelated networks. We have shown that in the infinite network limit, vertices in this network are almost surely equidistant if the relative number of interlinks is any finite number. Our approach can be applied to a number of other problems for networks of this sort, including the birth of a giant connected component, percolation, and others.

\section{Acknowledgments}

This work was partially supported by projects POCTI: FAT/46241/2002, MAT/46176/2002, FIS/61665/2004, and BIA-BCM $/ 62662 / 2004$, and also by project DYSONET-NEST/012911 and the ICT project SOCIALNETS, grant 217141. Authors thank A. V. Goltsev and A. M. Povolotsky for useful discussions.

\section{APPENDIX: STATISTICS OF THE CONFIGURATION MODEL}

For the sake of clarity, here we outline the Ztransformation (generating function) technique in application to the standard configuration model of an uncor- (a)

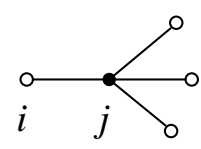

(b)

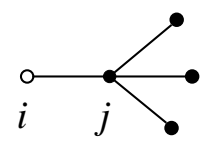

FIG. 3: The first (a) and the second (b) components of edge $(i j)$. Filled vertices belong to the components. The 0 -th component is empty.

related graph with a given degree distribution $\Pi(q)$. For more detail, see Refs. 8, 9, 10]. In simple terms, the configuration model [18, 19] is a maximally random graph with a given degree distribution. In graph theory it is also called a random graph with a given degree sequence.

Graph of size $N$ consists of a set of vertices $v_{i}, i=$ $1,2, \ldots, N$, connected by edges $e_{j i}$. An edge $e_{j i}$ exists if the adjacency matrix element $g_{j i}=1$. We start from the following distribution:

$$
\widetilde{\Pi}(q)=\frac{1}{2 L}\left\langle\sum_{j i=1}^{N} g_{j i} \delta_{K}\left(q_{j}-1-q\right)\right\rangle,
$$

where $\delta_{K}$ is the Kronecker symbol. This is the probability that a randomly chosen end of a randomly chosen edge in the graph has branching $q$. Alternatively, it may be considered as conditional probability for final vertex $j$ in a randomly chosen ordered pair $(j, i)$ to have degree $q+1$, provided vertices are connected by an edge. Obviously,

$$
\widetilde{\Pi}(q)=\frac{1}{2 L}\left\langle\sum_{j=1}^{N} q_{j} \delta_{K}\left(q_{j}-1-q\right)\right\rangle=\frac{q+1}{\bar{q}} \Pi(q+1),
$$

where $\bar{q} \equiv\langle q\rangle$ is the average degree of a vertex. In the Z-representation this relation takes the form:

$$
\widetilde{\phi}(x)=\sum_{q=0}^{\infty} \widetilde{\Pi}(q) x^{q}=\frac{1}{2 L}\left\langle\sum_{j=1}^{N} q_{j} x^{q_{j}-1}\right\rangle=\frac{\phi^{\prime}(x)}{\bar{q}} .
$$

Note that $\bar{q}=\phi^{\prime}(1)$.

Let the $n$-th component of the ordered pair $(j, i), C_{n, j i}$ be the following set of vertices. For any (ordered) pair of vertices $(j, i), C_{1, j i}$ is vertex $j$ if vertices are connected, else $C_{1, j i}=\varnothing$. For $n>1, C_{n, i j}$ is defined recursively as follows. If $g_{j i}=0$, all $C_{n, j i}=\varnothing$. Otherwise, in $C_{2, i j}$ there are also $q_{j}-1$ other vertices, connected to the vertex $j$, the third component $C_{3, i j}$ contains also all other vertices, connected with ones of the second component, and so on, see Fig. 3 .

In the thermodynamic limit $(N \rightarrow \infty)$ almost every finite $n$-th component of uncorrelated random graph is a tree. Then for the sizes (numbers of vertices) of the components, $M_{n, i j}=\left|C_{n, i j}\right|$ we have (assuming $g_{j i}=1$ ):

$$
M_{n, j i}=1+\sum_{k \neq i} M_{n-1, k j}
$$


with the initial condition $M_{1, j i}=1$. Due to the absence of correlations in the configuration model, $M_{n, k j}$ and $M_{n, l j}, k \neq l$, are independent equally distributed random variables. We define the distribution function of the $n$-th component of an edge as

$$
\begin{aligned}
p_{n}(M) & =\frac{1}{2 L} \sum_{j, i=1}^{N}\left\langle g_{j i} \delta\left(M_{n, i j}-M\right)\right\rangle \\
& =\frac{N(N-1)}{2 L}\left\langle g_{j i} \delta\left(M_{n, j i}-M\right)\right\rangle \\
& =\frac{1}{\left\langle g_{j i}\right\rangle}\left\langle g_{j i} \delta\left(M_{n, j i}-M\right)\right\rangle .
\end{aligned}
$$

It is more convenient to use the Z-transformation of this distribution:

$$
\psi_{n}(x)=\frac{1}{2 L}\left\langle\sum_{i, j=1}^{N} g_{i j} x^{M_{n, i j}}\right\rangle=\frac{1}{\left\langle g_{i j}\right\rangle}\left\langle g_{i j} x^{M_{n, i j}}\right\rangle .
$$

Substituting Eq. (A.4) into Eq. (A.6) and using Eq. A.3 gives

$$
\begin{aligned}
& \psi_{n}(x)=\frac{x}{2 L}\left\langle\sum_{j, i=1}^{N} g_{j i} \prod_{k \neq i,} \frac{1}{\left\langle g_{k j}\right\rangle}\left\langle g_{k j} x^{M_{n-1, k j}}\right\rangle\right\rangle \\
& =\frac{x}{2 L}\left\langle\sum_{j=1}^{N} q_{j}\left[\psi_{n-1}(x)\right]^{q_{j}-1}\right\rangle=x \widetilde{\phi}\left[\psi_{n-1}(x)\right] .
\end{aligned}
$$

Let $\mathcal{C}_{n, i}$ be the $n$-th component of vertex $v_{i}$. This component includes all vertices at distance $n$ or closer from vertex $v_{i}$. (The 0 -th component of a vertex is empty.) Due to the absence of loops (tree-like structure) we have the following relation for the size of $n$-th component of vertex $v_{i}, \mathcal{M}_{n, i}=\left|\mathcal{C}_{n, i}\right|$,

$$
\mathcal{M}_{n, i}=1+\sum_{j} M_{n-1, i j}
$$

So the $n$-th component size distribution

$$
P_{n}(\mathcal{M})=\frac{1}{N}\left\langle\sum_{i=1}^{N} \delta\left(\mathcal{M}_{n, i}-\mathcal{M}\right)\right\rangle
$$

is expressed in Z-representation as

$$
\Psi_{n}(x)=\frac{1}{N}\left\langle\sum_{i=1}^{N} x^{\mathcal{M}_{n, i}}\right\rangle=x \phi\left[\psi_{n-1}(x)\right] .
$$

The average sizes of subsequent $n$-th components are related through the following equations:

$$
\begin{aligned}
& \overline{\mathcal{M}}_{n}=\Psi_{n}^{\prime}(1)=1+\bar{q} \bar{M}_{n-1}, \\
& \bar{M}_{n}=1+\zeta \bar{M}_{n-1},
\end{aligned}
$$

where

$$
\zeta \equiv \widetilde{\phi}^{\prime}(1)=\frac{\phi^{\prime \prime}(1)}{\bar{q}}=\frac{1}{\bar{q}} \sum_{q=0}^{\infty} q(q-1) \Pi(q)=\frac{\left\langle q^{2}\right\rangle}{\bar{q}}-1,
$$

which is the mean branching. If $\zeta<1$, both $M_{n}$ and $\mathcal{M}_{n}$ have finite limits as $n \rightarrow \infty$. That is, the network has no giant connected component. If $\zeta>1$, a giant connected component exists.

Assuming $\psi_{n}=\psi_{n-1} \equiv \psi$ in Eq. (A.7), we obtain an equation for the distribution function of the sizes of edge's connected components,

$$
\psi(x)=x \widetilde{\phi}[\psi(x)],
$$

which implicitly defines $\psi(x)$. If $\zeta>1$, this equation has two solutions at $x=1$. One is $\psi(1)=1$, the other is some $\psi(1) \equiv t<1$,

$$
t=\widetilde{\phi}(t) .
$$

For any value of $\zeta, \psi_{n}(1)=1$. On the other hand, if $\zeta>1, \lim _{x \rightarrow 1-0} \lim _{n \rightarrow \infty} \psi_{n}(x)=t<1$. This is the probability that the connected component of a randomly chosen edge is finite. Then the probability that randomly chosen vertex belongs to a finite connected component of the graph is

$$
\sum_{q=0}^{\infty} \Pi(q) t^{q}=\phi(t)
$$

Therefore the number of vertices in the giant connected component in the thermodynamical limit is

$$
M_{\infty}=N[1-\phi(t)]
$$

One may find an intervertex distance distribution from the mean sizes of the $n$-th components of a vertex, see Ref. [8, 26] . The diameter $\bar{\ell}$ of the giant connected component, i.e., the distance between two randomly chosen vertices, is obtained from the relation $\zeta \sim M_{\infty} \sim N$. So, if the second moment of the degree distribution is finite,

$$
\bar{\ell} \cong \frac{\ln a N}{\ln \zeta},
$$

where $a$ is some number of the order of 1 . For more straightforward calculations, see Refs. [10, 27].
[1] R. Albert and A.-L. Barabási, Statistical mechanics of complex networks, Rev. Mod. Phys. 74, 47 (2002).
[2] S. N. Dorogovtsev and J. F. F. Mendes, Evolution of 
networks, Adv. Phys. 51, 1079 (2002); Evolution of Networks: From Biological Nets to the Internet and $W W W$ (Oxford University Press, Oxford, 2002).

[3] M. E. J. Newman, The structure and function of complex networks, SIAM Review 45, 167 (2003).

[4] R. Pastor-Satorras and A. Vespignani, Evolution and Structure of the Internet: A Statistical Physics Approach (Cambridge University Press, Cambridge, 2004).

[5] S. Boccaletti, V. Latora, Y. Moreno, M. Chavez, and D.U. Hwang, Complex Networks: Structure and Dynamics, Phys. Reports 424, 175 (2006).

[6] G. Caldarelli, Scale-Free Networks (Oxford University Press, Oxford, 2007).

[7] S. N. Dorogovtsev, A. V. Goltsev, and J. F. F. Mendes, Critical phenomena in complex networks, arXiv:0705.0010 [cond-mat].

[8] M. E. J. Newman, S. H. Strogatz, and D. J. Watts, Random graphs with arbitrary degree distributions and their applications, Phys. Rev. E 64, 026118 (2001).

[9] M. E. J. Newman, Random graphs as models of networks, in Handbook of Graphs and Networks: From the Genome to the Internet, eds. S. Bornholdt and H. G. Schuster (Wiley-VCH, Berlin, 2002), p. 35.

[10] S. N. Dorogovtsev, J. F. F. Mendes, and A. N. Samukhin, Metric structure of random networks, Nucl. Phys. B 653, 307 (2003).

[11] One can easily understood the vertex equidistance in typical infinite small worlds in the following way. The mean number of $\ell$-th nearest neighbors of a vertex in small worlds usually grows with $\ell$ as $\zeta$, where $\zeta$ is the mean branching coefficient, and $\ell \lesssim \bar{\ell}(N)$. This leads to the following estimate for the intervertex distance distribution: $\mathcal{P}(\ell, N) \propto \zeta \theta(\bar{\ell}(N)-\ell)$. In addition, one must take into account normalization, which readily gives $\mathcal{P}(\ell, N) \rightarrow \delta(\ell-\bar{\ell}(N))$ as $N \rightarrow \infty$.

[12] P. Pollner, G. Palla, and T. Vicsek, Preferential attachment of communities: the same principle, but a higher level, Europhys. Lett. 73, 478 (2006).

[13] K. Suchecki and J. A. Holyst, First order phase transition in Ising model on two connected Barabási-Albert networks, arXiv:0802.1499

[14] R. K. Pan and S. Sinha, Modular networks emerge from multiconstraint optimization, Phys. Rev. E 76, 045103 (R) (2007); The small world of modular networks, arXiv:0802.3671

[15] A. Galstyan and P. Cohen, Cascading dynamics in modular networks, Phys. Rev. E 75, 036109 (2007).

[16] M. E. J. Newman and J. Park, Why social networks are different from other types of networks, Phys. Rev. E 68, 036122 (2003).

[17] J.-L. Guillaume and M. Latapy, Bipartite structure of all complex networks, Information Processing Letters 90, 215 (2004).

[18] B. Bollobás, Eur. J. Comb. 1, 311 (1980).

[19] E. A. Bender and E. R. Canfield, J. Combin. Theory A, 24, 296 (1978).

[20] M. Molloy and B. Reed, Random Structures and Algorithms 6, 161 (1995).

[21] M. Molloy and B. Reed, Combinatorics, Probability and Computing 7, 295 (1998).

[22] D. Fernholz and V. Ramachandran, Cores and connectivity in sparse random graphs, UTCS Technical Report TR04-13, 2004.

[23] S. N. Dorogovtsev, A. V. Goltsev, and J. F. F. Mendes, $k$ core organization of complex networks, Phys. Rev. Lett. 96, 040601 (2006); $k$-core architecture and $k$-core percolation on complex networks, Physica D 224, 7 (2006); A. V. Goltsev, S. N. Dorogovtsev, J. F. F. Mendes, kcore (bootstrap) percolation on complex networks: Critical phenomena and nonlocal effects, Phys. Rev. E 73, 056101 (2006).

[24] J.I. Alvarez-Hamelin, L. Dall'Asta, A. Barrat, and A. Vespignani, $k$-core decomposition: a tool for the visualization of large scale networks, Adv. Neural Information Processing Systems Canada 18, 41 (2006).

[25] S. Carmi, S. Havlin, S. Kirkpatrick, Y. Shavitt, and E. Shir, New model of Internet topology using $k$-shell decomposition, PNAS 104, 11150 (2007).

[26] R. Cohen and S. Havlin, Scale-free networks are ultrasmall, Phys. Rev. Lett. 90, 058701 (2003).

[27] A. Fronczak, P. Fronczak, and J. A. Holyst, Average path length in uncorrelated random networks with hidden variables, Phys. Rev. E 70, 056110 (2004). 